\title{
MODELLING THE WIND ECLIPSES IN WR+O BINARIES: THE QUALITATIVE PICTURE
}

\author{
Gloria Koenigsberger \\ Instituto de Astronomía, UNAM
}

Lawrence H. Auer

Los Alamos National Laboratory, Los Alamos, NM

\begin{abstract}
The phase dependent profile variations due to wind eclipses in $\mathrm{WR}+\mathrm{O}$ binary systems are shown to be a means of establishing the WR wind structure. The results of two model calculations are presented which indicate that there are important qualitative differences between the profile variatons which will be observed in slowly -and rapidly- accelerating winds.
\end{abstract}

\section{INTRODUCTION}

Recent developments in the treatment of radiative transfer in the winds of WR stars now provide a means of diagnosis of the intrinsic properties of these stars (Schmutz and Hammann, and Wessolowski 1989; Hillier, 1988). However, one indeterminate quantity in these analyses which introduces uncertainties in the results is the distance scale within the wind; that is, the location of the gas forming the spectra that are being modelled cannot be related to the center of the star (Hummer, 1990). Binary systems in which the orbital parameters are well determined provide such a "yardstick" and the line profile variations which occur as a result of the wind eclipse of the companion O-star by the WR wind can be used to derive information on the structure of the WR wind. With a broad spectral coverage, it is possible in principle to derive the velocity and ionization structure (c.f. Koenigsberger 1990).

\section{WIND ECLIPSES AND PROFILE VARIATIONS}

As first suggested by Munch (1950), when the O-star companion to a WR star is behind some portion of the WR wind, its continuum radiation is absorbed by this wind at line wavelengths, enhancing the $P$ Cyg-type absorptions present in the spectrum. In addition, however,as modelled by Khaliullin and Cherepashchuk (1976) the strength of the emission component is reduced due to absorption by gas traveling with a velocity component 
towards the O-star. These wind eclipses give rise to periodic profile variations which are observed to be stronger in the UV that in the optical spectra (Koenigsberger and Auer 1985 and refs. therein).

Given the orbital parameters of the binary system, one can calculate the impact parameter of the ray along the line-of sight to the O-star as a function of the orbital phase. If one assumes that the phase dependent variations result exclusively from the wind eclipse mechanism, then it is possible to associate the observed profile variations with changes in the wind structure along each ray as a function of impact parameter. This allows a contraint to be placed on the physical position within the wind of the absorbing/emitting gas, and on the manner in which the velocity and density structures change with distance from the WR core. Thus, the change in the P Cygni structure of the line, from one orbital phase to another, provides information on the change in the structure of the wind from one impact parameter to another.

In order to be able to interpret the observations it is necessary to model the radiative transfer. In the simplest approximation, this just requires ray tracing, and a code which interactively permits this analysis is now available. The program treats the full velocity dependence of the radiation transfer along the line of sight to the O-star. It does not assume the transsonic or "Sobolev" limit, and can be used to estimate empirically the velocity and the density structure of the WR wind. The details of the calculations and a grid of models will be presented elsewhere.

One of the basic questions which may be addressed with this method is the velocity structure within the wind.

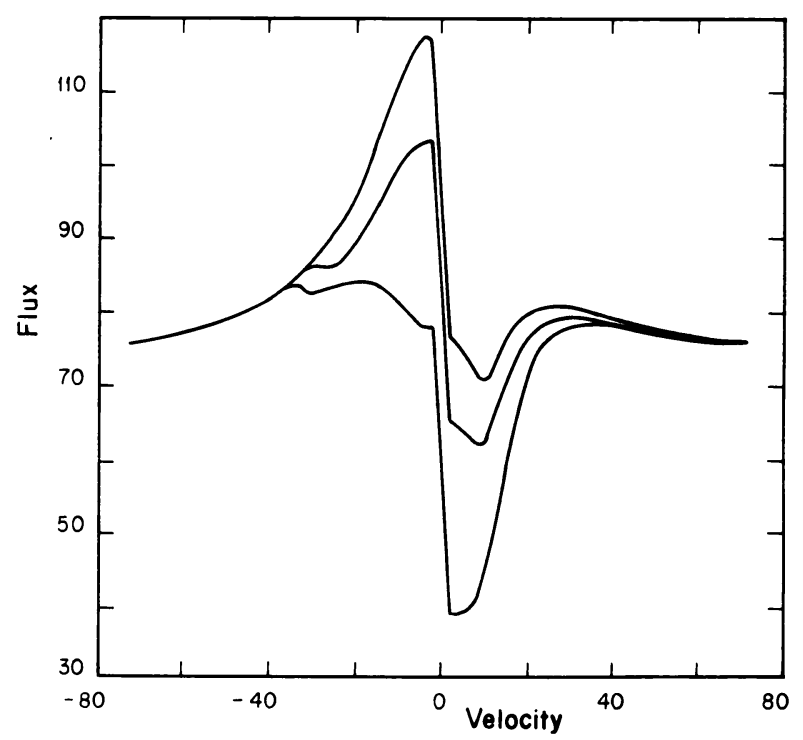

Figure 1: Results of a model calculation showing the phase dependent profile variations which result from wind eclipse for the case of a linear velocity law. The three curves correspond to impact parameters (from top to down) $p / r_{*}=9.9,5.9$ and 3.1. In this reference frame positive velocities correspond to gas moving towards the observer. 
In Figure 1 we illustrate the predicted profile variations for the case of a linear velocity law, $v(r)=v\left(r / r_{*}\right)$ out to 20r*. This is a "slow" velocity law, in which terminal speeds are achieved far from the WR core, and the density falls off as $r^{-3}$. In Figure 2 we illustrate the predicted variations for a velocity law of the form $v(r)=v_{\infty}\left(1-r / r_{*}\right)^{\beta}$, in this case with $\beta=0.5$. This is a very fast velocity law, since terminal speeds are achieved within less that a couple of stellar radii. In both calculations we have taken the opacity $\chi(r)=F(r) / v(r) r^{-2}$ with $F(r)=$ constant throughout, an O-star to WR-star continuum luminosity ratio of 1 , an $O$-star radius of $3 r_{*}$, and an orbital separation of $10 r_{*}$.

Disregarding the intrinsic shape of the profiles, but concentrating only on the nature of the phase-dependent variations, there are two major differences which emerge from a comparison of Figures 1 and 2:

1. There is a "narrow", high velocity absorption spike which becomes stronger as a function of decreasing impact parameter in the case of the $\beta=0.5$ velocity law which is completely absent in the case of a linear velocity law. This spike becomes less prominent as $\beta$ becomes larger, but is present even at $\beta=3$, and results from the large column density of gas traveling at constant velocity and which is projected onto the O-star.

2. The profile changes are much more prominent in the case of a linear velocity law and occur at larger impact parameters than for the $\beta=0.5$ law; i.e., at $p=3.09 r *$ the emission component in Fig. 1 has been completely overwhelmed by the absorption. This is not the case in Fig. 2 because of the (Sobolev) optical depth falls off more rapidly due to the larger velocity gradients along the line of sight to the O-star.

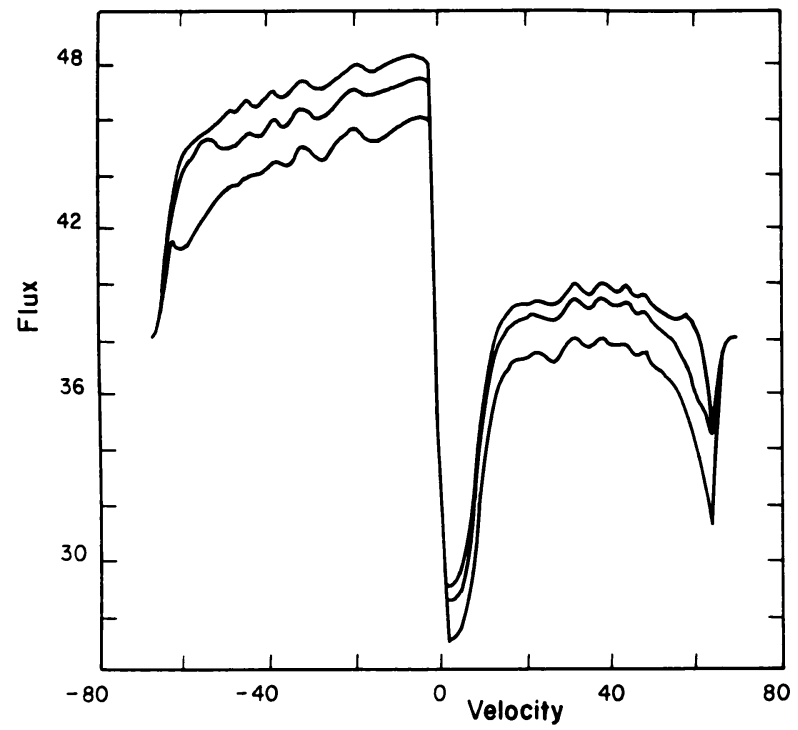

figure 2: same as Fig. 1 but for a $\beta=0.5$ velocity law. 


\section{CONCLUSIONS}

Given the above results, it would appear to be relatively simple to descriminate between a fast and a slow velocity law based on a comparison of observed profile changes with model calculations. In practice, however, the picture is not that simple. In the above examples we have not taken into account the presence of emission lines arising in a wind associated with the $\mathrm{O}$-star, nor the effects introduced by a possible wind-wind collision. In addition, local fluctuations, such as those produced by inhomogeneities in the WR wind, will introduce uncertainties in the interpretation. These problems, however, are soluble or may be avoided by chosing a binary system with a late-type Main Sequence companion and by averaging the observations over several orbital cycles. Thus, a quantitative application of the use of wind eclipses may provide the tool required for determining the wind structure in those WR stars where it is applicable.

\section{REFERENCES}

Hillier, J.D. 1988, Ap. J., 327, 822.

Hummer, D. 1990, private communication.

Khaliullin, Kh. and Cherepashchuk, A.M. 1976, Soviet Astron., 20, 186.

Koenigsberger, G. 1990, Astro. Astrof., in press.

Koenigsberger, G. and Auer, L.H. 1985, Ap. J., $297,255$.

Munch, G. 1950, Ap. J., 112, 266.

Schmutz, W. and Hamman, W.-R., Wessolowski, V. 1989, Astron. Astrof., 210. 236.

\section{DISCUSSION}

Owocki: What are the relative $\dot{M}$-values of the $\mathrm{O}$ and WR stars? This will indicate the scale of the interaction zone - if $\dot{M}_{W R} \gg \dot{M}_{O}$ then this interaction will be confined to very near the $\mathrm{O}$ star.

Koenigsberger: The ratio of total wind momentum in V444 Cyg is 30 (Pollock, Blondin and Stevens, this symposium).

Leitherer: You find a relatively shallow velocity law with accerelation up to $\sim 5 R_{*}$. On the other hand, we heard yesterday from Hamann and Hillier that they find optimum fits to optical recombination lines and UV resonance lines with a very steep CAK-type law. Do you have an explanation for this difference?

Koenigsberger: My analysis does not rule out a rapidly accelerating zone near the WR core. At this stage all I can say is that significant acceleration must still be occurring at a distance of several stellar radii.

Shylaja: There is a general problem in fixing the continuum of WR energy distribution. How was this avoided?

Koenigsberger: I always try to use ratios of spectra: the spectrum of the system taken at quadrature is used in the denominator and that at other phases in the numerator. 